\title{
Review of fluorescein angiograms performed in one
}

year

W N Wykes, S J Livesey
Table 2 Indication for FFA

\begin{tabular}{lrrr} 
& UHW & St Woolos & Total \\
\hline Maculopathies: & 111 & 54 & 165 \\
senile macular degeneration & 1 & 5 & 6 \\
central serous retinopathy & 18 & 4 & 22 \\
cystoid macular oedema & 1 & 4 & 5 \\
Foerster-Fuchs maculopathy & 1 & 2 & 3 \\
preretinal gliosis & 5 & 1 & 6 \\
retinal pigment epithelial detachment & 0 & 1 & 1 \\
choroiditis & 3 & 1 & 4 \\
circinate retinopathy & 6 & 1 & 7 \\
aneurysm & 3 & 1 & 4 \\
hereditary maculopathy & 5 & 3 & 8 \\
unexplained poor vision & 27 & 23 & 50 \\
Retinal vascular disease: & 10 & 8 & 18 \\
diabetes & 0 & 1 & 1 \\
venous occlusion & 1 & 0 & 1 \\
periphlebitis & & 3 & 7 \\
hypertension & 4 & 3 & 1 \\
Optic disc: & 1 & 0 & \\
papilloedema & 13 & 7 & 20 \\
drusen & 1 & 0 & 1 \\
Ocular tumours: & 211 & 119 & 330 \\
malignant melanoma & & & \\
metastases & &
\end{tabular}

In all cases $20 \%$ sodium fluorescein was used intravenously. No serious side effects were reported.

Fluorescein angiography has been available as an ophthalmic conditions since its introduction by Novotny and Alvis in $1961 .^{1}$ Previous studies have suggested the main area in which it is helpful. ${ }^{2-6}$ These are: macular disease; retinal vascular disease; the optic disc; ocular tumours. However, few papers have tried to quantify its value..$^{7-9}$

In this study we assess the reason for performing the test and its usefulness in the management of the patient concerned.

\section{Material and method}

All the fundus fluroescein angiograms (FFAs) performed in 1986 in two centres, the University Hospital of Wales, Cardiff (catchment: 542000 ) and St Woolos Hospital, Newport, Gwent (catchment: 440000 ) were examined to ascertain the reasons for requesting the test, the distribution of conditions investigated, and the outcome - that is, whether the FFA altered management and if so what the final visual outcome was.

Three hundred and seventy four FFAs on 330 patients are included in the study. Thirty four patients underwent more than one FFA, totalling 78 between them (Table 1).

\section{Results}

\section{INDICATION FOR FFA}

Maculopathy was, as expected, the commonest indication, particularly to ascertain the presence and location of disciform maculopathy. Diabetic retinopathy was also a fairly common indication. Table 2 lists the indications: 231 patients were investigated for maculopathy other than diabetic maculopathy, of whom 165 had clinical evidence of senile macular degeneration, 58 were thought to have other macular pathology, and eight were thought to have normal fundi, with unexplained poor visual acuity.

Fifty patients were diabetic, and the remaining 50 were investigated for malignant melanoma, venous occlusion, papilloedema, periphlebitis, disc drusen, and choroidal metastases.

Of the 34 patients who underwent more than one FFA 30 had a disciform macular degeneration. Of these, 26 had two FFAs, three had three FFAs and one had eight FFAs. Of the remaining four patients one had a malignant melanoma, treated by laser (three FFAs), one had dry senile

Table 1 Fluorescein angiograms performed

\begin{tabular}{llllll}
\hline Hospital & $\begin{array}{l}\text { FFA } \\
\text { performed }\end{array}$ & $\begin{array}{l}\text { Notes not } \\
\text { found }\end{array}$ & $\begin{array}{l}\text { FFA in } \\
\text { study }\end{array}$ & $\begin{array}{l}\text { Patients } \\
\text { in study }\end{array}$ & $\begin{array}{l}\text { Patients with } \\
\text { repeat FFA }\end{array}$ \\
\hline UHW, Cardiff & 279 & 44 & 235 & 211 & 22 \\
St Woolos, & 147 & 8 & 139 & 119 & 12 \\
$\begin{array}{l}\text { Newport } \\
\text { Total }\end{array}$ & 426 & 52 & 374 & 330 & 34 \\
\hline
\end{tabular}
maculopathy (two FFAs), one had choroiditis (two FFAs), and one had a venous occlusion (two FFAs).

\section{RESULTS OF FFAS}

Maculopathy. FFAs were performed on 165 patients with the clinical diagnosis of age related macular degeneration. Seventy (42\%) were 
Table 3 Macular degeneration

Maculopathy:
70 dry maculopathy
18 misdiagnosed
77 disciform maculopathy: 57 subfoveal, no treatment
20 treatable, laser
Total 165

shown to have dry maculopathy, 18 (11\%) had been misdiagnosed, and $77(47 \%)$ had subretinal neovascularisation, of whom 57 had untreatable subfoveal neovascularisation while the other 20 (12\%) received laser treatment (Table 3).

In the treated group the average initial vision was $6 / 24$ and the final vision $6 / 36$. Three patients were improved when last seen, two remained static, and the rest deteriorated. Follow-up time ranged from one to 18 months. One patient maintained a visual acuity of $6 / 18$ for 15 months before deteriorating, but the rest deteriorated within three months of treatment.

Table 4 shows the other maculopathies, excluding diabetic maculopathy. One patient thought to have cystoid macular oedema was found to have central serous retinopathy, and another had a microaneurysm. Three cases of circinate retinopathy were examined, one due to aneurysms and treated with laser, and one with a leaking site close to the macula for which treatment with laser was offered but was declined.

One case of choroiditis had changes consistent with presumed ocular histoplasmosis syndrome; one patient with suspected hereditary macular degeneration was shown to have a gross pigment defect centrally in both eyes.

Eight patients with unexplained poor visual acuity underwent fluorescein angiography to eliminate any maculopathy, but all were found to be normal, suggesting that FFA is unhelpful in the absence of clinically observable changes.

Diabetic retinopathy. The reasons for investigation in the 50 diabetic patients included in the study are shown in Table 5.

Following angiography 10 of the 25 patients with maculopathy received laser treatment. All the patients with circinate retinopathy received laser treatment, but only three showed leakage of fluorescein. Six of the nine patients with possible preproliferative disease showed sufficient

Table 4 Outcome of other maculopathies

\begin{tabular}{lrcc}
\hline Clinical diagnosis & No & Confirmed & Not confirmed \\
\hline CMO & 22 & 12 & 10 \\
CSR & 6 & 2 & 4 \\
RPE detachment & 6 & 3 & 3 \\
Foerster-Fuchs & 5 & 1 & 4 \\
Isolated aneurysm & 5 & 3 & 2 \\
Preretinal gliosis & 3 & 2 & 1 \\
Circinate retinopathy & 3 & 3 & - \\
RPE defect & 3 & 3 & - \\
POHS & 1 & 1 & - \\
Hereditary macular degeneration & 1 & 1 & -
\end{tabular}

$\mathrm{CMO}=$ cystoid macular oedema $. \mathrm{CSR}=$ central serous retinopathy. $\mathrm{RPE}=$ retinal pigment epithelium. $\mathrm{POHS}=$ presumed ocular histoplasmosis syndrome.

Table 5 Diabetic retinopathy

\begin{tabular}{lcllll}
\hline Clinical diagnosis & No & Confirmed & Revised & Unhelpful & Laser \\
\hline Maculopathy & 25 & 10 & - & 15 & 10 \\
Circinate & 10 & 3 & - & 7 & 10 \\
Preproliferative changes & 9 & 6 & 2 & 1 & 6 \\
Disc new vessels & 6 & 5 & 1 & - & 5 \\
\hline
\end{tabular}

changes to warrant laser treatment. Disc new vessels were confirmed in five of six patients, and they received a laser panretinal ablation to the affected eye. However, in two of these patients the new vessels were recorded as obvious clinically and were readily identifiable on colour fundus photograph alone, so that, as with the 10 cases of circinate retinopathy, FFA did not influence the management of the patient.

Vascular occlusion. Eighteen patients with venous occlusion underwent FFA. Two of these were for retinal vein occlusion with possible capillary closure, neither of which required laser treatment; 11 were for branch vein occlusion, some with secondary maculopathy; six received laser treatment; three patients with branch vein occlusions were misdiagnosed as disciform macular degeneration, two of whom received laser treatment; two patients with collaterals at the optic disc underwent FFA to ensure that these were not new vessels.

One patient with Crohn's disease was noted to have perivenous sheathing. An FFA did not show any evidence of periphlebitis.

Tumour. Twenty patients with pigmented fundus lesions were investigated. Twelve were shown to have choroidal naevi, one had a serous retinal detachment with no definite underlying mass, and seven had appearances typical of malignant melanoma. Three of these patients underwent enucleation, with subsequent confirmation of the diagnosis. Two were managed conservatively (by observation), one received radiotherapy, and one had a small malignant melanoma which was treated with laser. This patient has required several further applications of the laser.

In one patient a choroidal metastasis was confirmed.

Optic disc. Seven patients underwent FFA for suspected papilloedema. Two were confirmed and three appeared completely normal. One, with reduced vision, had a genuinely swollen left disc and a diagnosis of papillitis was made. One with normal vision and a unilateral swollen disc was shown on FFA to have a juxtapapillary lesion, by which time the visual acuity had fallen. A diagnosis of juxtapapillary choroiditis was made, and the patient was started on systemic steroids, with full recovery of visual acuity.

Three patients were thought to have disc drusen, which were confirmed in two of them.

Misdiagnosed conditions. Eighteen patients with an original diagnosis of disciform macular degeneration were found after FFA to have the following: pigment epithelial detachments (five patients), pigmentary changes associated with preretinal gliosis (five patients), small branch retinal vein occlusions (six patients), microaneurysm (one patient), and naevus (one patient).

\section{Discussion}

This survey shows that fluorescein angiography is used in these units mainly as expected, with the single largest indication being macular degeneration. It must be remembered that the spectrum of disease shown does not represent that seen in the clinic as many cases can be treated on clinical grounds alone. 
Over $40 \%$ patients investigated for possible disciform maculopathy were found to have dry changes only, and it should be possible by careful clinical assessment to reduce this number. Part of the reason for this was that contrary to unit policy some of the FFAs had been requested by doctors other than consultants and senior registrars. Review of the visual acuity and colour fundus photographs suggested that the unnecessary FFAs had often been requested by more junior members of staff, and it is recommended that FFAs should only be requested by more experienced ophthalmologists.

As age related maculopathy was the commonest reason for requesting an FFA, the clinical course and final visual outcome were examined in a little more detail to give some idea of the 'usefulness' of the investigation in this condition. The results of treatment of the patients with apparently treatable disciform maculopathy were disappointing.

It has been suggested that patients with age related maculopathy should be seen urgently, particularly if the history of visual disturbance is short and the visual acuity is good. ${ }^{10}$ Unfortunately this information is not always available on referral letters, and this raises the question whether all cases referred with possible macular degeneration should be seen urgently or routinely in units where the routine waiting time for new appointments may be several months. This paper did not set out to answer that question, but it does show that when disciform lesions which do not involve the fovea on FFA are treated the visual results are poor. Those patients with maculopathy investigated by fluorescein angiography are a small percentage of those seen in the clinic, as many patients are obviously not treatable on clinical grounds. Of 163 patients investigated 20 received treatment, five of whom maintained or improved their visual acuity and one of whom improved for more than three months. Is it justifiable to displace outpatient appointments for patients with known treatable conditions such as cataract for such a low yield in terms of improved visual acuity? One factor which does help to decide urgency of appointment is the inclusion of visual acuity on the referral letter, and general practitioners should be encouraged at every opportunity to provide this information.

The second largest group investigated was that of patients with diabetic retinopathy. Fluorescein angiography was useful in differentiating between cystoid macular oedema and ischaemic maculopathy, and therefore whether laser therapy was indicated. On the whole, it proved unhelpful in the diagnosis and treatment of circinate retinopathy, but in one case two leaking sites were found on FFA rather than the one obvious clinically.

Previously it has been reported that the most commonly missed diagnoses which fluorescein angiography has corrected are branch retinal vein occlusion, chronic central serous choroidopathy, and retinal artery aneurysm with subretinal or subhyaloid haemorrhage." This study found six cases of branch retinal vein occlusion and one aneurysm, as well as several cases of pigment epithelial detachment and preretinal gliosis, all clinically misdiagnosed as disciform macular degeneration.

In conclusion, although it is still very difficult to quantify the clinical value of fluorescein angiography, it is possible to make an attempt in conditions such as macular degeneration, and in this age of medical audit some attempt to assess the value and limitations of a relatively costly and time consuming investigation (which bears some risk to the patient) must be made. The study does show the circumstances in which fluorescein angiography is useful and where unnecessary angiograms could be reduced. Several patients with dry macular degeneration need not have had FFAs. None of the patients with unexplained poor visual acuity showed any abnormality, suggesting that it is not very useful in such cases, and only one of the five FFAs performed for circinate retinopathy influenced management. If one could cut down on unnecessary FFAs, this would reduce the waiting time for the investigation.

The authors thank the consultant ophthalmologists at the University Hospital of Wales and St Woolos's Hospital for allowing data sity Hospital of Wales and St Woolos's Hospital for allowing data from their patients to be studied, and Dr
Glasgow, for his help in preparing the paper.

1 Novotny R, Alvis DL. A method of photographing fluorescence in circulating blood in the human retina. Circulation escence in circula

2 Ffytche TJ, Shilling JS, Chisholm IH, Federman JL. Indications for fluorescein angiography in disease of the ocular fundus: a review. $\mathcal{F} R$ Soc Med 1980; 73: 362-5

3 Norton EWD. Fluorescein angiography: twenty years later. Trans Ophthalmol Soc UK 1981; 101 : 229-33.

4 Blacharski PA. Twenty-five years of fluorescein angiography. Arch Ophthalmol 1985; 103: 1301-2.

5 Wilkinson CP. Limitation and over utilisation of angiographic services. Ophthalmology 1986; 93: 401-4.

6 Singerman LJ. Fluorescein angiography; practical role in the office management of macular diseases. Ophthalmology 1986; 93: 1029-15.

7 Jain LS, Dogra MR, Gupta A, Dhir SP. Value of fluorescein angiography in clinical ophthalmology. Ind $\mathcal{F}$ Ophthalmol angiography in clin

8 Garg SP, Tewari HK, Khosla PK. Role of fluorescein angiography in choroiditis. Ind $\mathcal{F}$ Ophthalmol 1983; 31: 5523.

9 D'Ettore MD, Nardini M, Menchini U, et al. Fluorescein retinal angiography in the early diagnosis of optic disc oedema. Eur Neurol 1981; 20: 401-10.

10 Bird AC. Laser photocoagulation of senile macular degeneration. BMF 1983; 286: 1001 .

11 Irvine AR. The diagnoses most commonly missed by ophthalmologists referring patients for fluorescein angiography. Ophthalmolgoy 1986; 93: 1216-21. 